\section{Screening for alcohol misuse in sexual health clinics}

\author{
Keith Radcliffe, Nicola Thorley
}

In this issue of the journal, Crawford et $a l^{1}$ make a useful contribution to the ongoing debate regarding whether alcohol screening and brief intervention for excessive drinkers should be delivered within sexual health clinics.

Alcohol misuse in the UK is responsible for considerable morbidity and mortality, as well as financial cost to the National Health Service, estimated at $£ 2.9$ billion in 2008-2009. ${ }^{2}$ The National Institute for Health and Care Excellence advises that all sexual health clinics should routinely screen patients for excessive alcohol use and deliver a brief alcohol behavioural intervention $(\mathrm{ABI})$ when problem dinking is identified. ${ }^{2}$

A commonly held view is that intoxication due to alcohol directly causes unsafe sexual behaviour, which in turn leads to higher rates of sexually transmitted infections (STI) and unwanted pregnancies, but the evidence does not support this simplistic model. A substantial body of evidence has established that alcohol misuse is associated with both high-risk sexual behaviour, including unprotected sex with multiple partners, underage or early sexual intercourse and emergency contraception use, and with negative sexual health outcomes. ${ }^{3}$ A systematic review found that eight of 11 relevant studies showed a significant association between alcohol misuse and STI. ${ }^{4}$ However, this association does not prove causation and may be due to either confounding bias (an underlying common cause) or reverse causation. Reviews of the evidence have failed to establish a causal relationship between use of substances, including alcohol, and highrisk sexual behaviour. ${ }^{5}$ However, there is evidence that an underlying excitementseeking personality type is a common causal factor for both alcohol misuse and high-risk sexual behaviour. ${ }^{6}$

Self-completion screening tools have been validated for detecting problem drinking in healthcare settings (eg, Alcohol Use Disorders Identification Test (AUDIT), AUDIT-C, Fast Alcohol Screening Test

Department Sexual Health, University Hospitals Birmingham, Birmingham, UK

Correspondence to Dr Keith Radcliffe, Department Sexual Health, University Hospitals Birmingham, Birmingham B4 6DH, UK; Keith.Radcliffe@uhb.nhs.uk
(FAST), Paddington Alcohol Test (PAT)), which only take a few (3-5) minutes to complete. Alcohol screening in sexual health clinics has been shown to be acceptable to patients. ${ }^{78}$ Reported rates of hazardous drinking (28\%-34\%) are higher among sexual health clinic attendees than in the general population $(26 \%) .{ }^{8} \quad 9$ Onward referral of hazardous drinkers to specialist alcohol services has been shown not to be an acceptable strategy in the sexual health setting, suggesting that any intervention will need to be delivered immediately in the clinic. ${ }^{8}$

A meta-analysis showed that brief advice for excessive alcohol consumption is effective at reducing alcohol misuse in patients across a range of medical settings other than sexual health. ${ }^{10}$ Of note, a systematic review showed that just being in the control group in intervention studies produced a benefit, a finding that is also apparent in randomised clinical trials (RCTs) conducted within the sexual health setting. 71112

Although no systematic reviews or meta-analyses have been published assessing $\mathrm{ABI}$ in the sexual health setting, three RCTs conducted in sexual health clinics in Sydney, London and Aberdeen showed that screening for excessive alcohol consumption is acceptable in sexual health clinics and identifies high rates of hazardous drinking ( $\mathrm{S}$ Baguley, personal communication, 2014). ${ }^{1} 712$ An important finding from two of these RCTs is that screening and simple feedback alone can lead to a significant reduction in AUDIT scores. ${ }^{7}{ }^{12}$ None of these RCTs demonstrated that ABI is more effective in reducing alcohol consumption than simple feedback (S Baguley, personal communication, 2014). ${ }^{1712}$ The methods, duration and timing of ABI delivery differed between the three RCTs. The Sydney study involved delivery of ABI (5-10 min duration) at the same appointment as screening and $74 \%$ of patients found this acceptable. Substantial reductions in both AUDIT scores and alcohol consumption were seen in both intervention and standard care arms. ${ }^{7}$ In contrast, the intervention group in the Crawford et al study consisted of feedback from the treating clinician (up to $3 \mathrm{~min}$ duration), written information and an offer of a longer appointment (up to $30 \mathrm{~min}$ duration) with an alcohol health worker (AHW). The authors concluded that universal screening and brief advice for excessive alcohol use did not result in significant reductions in alcohol consumption or provide a cost-effective use of resources, but it is important to note that only $20 \%$ of excessive drinkers recruited to the intervention arm actually attended for the AHW appointment, which may explain the lack of benefit in the intervention arm. The RCT in the Aberdeen sexual health clinic consisted of three study arms and compared ABI (2-5 min duration), weekly motivational text messaging (SMS) and simple feedback. ${ }^{12}$ Overall, there was no difference in efficacy between the intervention and control arms. However, on subgroup analysis the study demonstrated that ABI was more effective than SMS in under 25 year olds, but that SMS was superior to $\mathrm{ABI}$ in over 25 year olds. Simple screening and feedback was effective in reducing AUDIT scores in women, but was only effective in men aged over 25 years ( $S$ Baguley, personal communication, 2014). ${ }^{12}$ This study raises the question as to whether interventions may need to be tailored according to age and gender in order to be effective. In conclusion, in the sexual health setting simple feedback alone can lead to reductions in AUDIT scores but ABI has not shown superiority over simple feedback of results. As the observed reduction in hazardous drinking was not associated with a reduction in either risky sexual behaviour or adverse sexual health outcomes, these studies do not support a direct causal connection between hazardous drinking and poor sexual health. ${ }^{112}$

There has been a movement in health policy towards introducing alcohol screening in sexual health clinics, which aims to reduce alcohol consumption and alcoholrelated harm, but there is no evidence that this will lead to improvements in the sexual health of patients. There is evidence that alcohol screening is feasible and acceptable within the sexual healthcare setting, but there is no evidence that $\mathrm{ABI}$ is more effective at reducing alcohol consumption than simple screening and feedback or that it reduces sexual illhealth. Simple screening and feedback may be a clinical and cost-effective strategy for managing hazardous drinkers within sexual health clinics, and more research in this area is warranted.

Health policymakers need to decide whether the role of sexual health clinics is to improve the sexual health of patients or whether their responsibility should be extended to health promotion activities that might benefit the general health of attendees, but which will not improve their sexual health. The latter approach would 
mean that resources would need to be diverted away from current sexual health priorities in order to provide alcohol screening and deliver ABI within the clinic. If it is accepted that sexual health clinics should be responsible for general health promotion, should their remit then logically be expanded to incorporate screening and interventions for obesity, smoking and physical inactivity as well?

Finally, alcohol misuse is a society-wide problem, and any health policy that hopes to reduce its consequences will also need to be society-wide. It should be remembered that there is strong evidence for the effectiveness of increasing the price of alcohol, and restricting advertising and availability, in terms of reducing the considerable harm that results from the consumption of excess alcohol. ${ }^{2}$

Competing interests None.

Provenance and peer review Commissioned; internally peer reviewed.

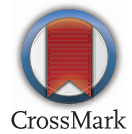

To cite Radcliffe K, Thorley N. Sex Transm Infect 2015:91:4-5.

Accepted 16 October 2014

Published Online First 4 November 2014

\section{C) Linked}

http://dx.doi.org/10.1136/sextrans-2014-051561

Sex Transm Infect 2015;91:4-5.

doi:10.1136/sextrans-2014-051881

\section{REFERENCES}

1 Crawford MJ, Sanatinia R, Barrett B, et al. The clinical and cost-effectiveness of brief advice for excessive alcohol consumption among people attending sexual health clinics: a randomised control trial. Sex Transm Infect 2015;91:37-43.

2 National Institute for Health and Clinical Excellence (NICE). NICE PH24 guidelines: Alcohol-use disorders -preventing harmful drinking. NICE, 2010. http:/l www.nice.org.uk/guidance/PH24 (accessed 15 Oct 2014)

3 Aicken C, Nardone A, Mercer C, et al. Alcohol misuse, sexual risk behaviour and adverse sexual health outcomes: evidence from Britain's national probability sexual behaviour surveys. J Public Health 2011;33:262-71

4 Cook RL, Clark DB. Is there an association between alcohol consumption and sexually transmitted diseases? A systematic review. Sex Transm Dis 2005;32:156-64.

5 Rashad I, Kaestner R. Teenage sex, drugs and alcohol use: problems identifying the cause of risky behaviours. J Health Econ 2004;23:493-503.

6 Justus A, Finn P, Steinmetz J. The influence of traits of disinhibition on the association between alcohol use and risky sexual behaviour. Alcohol Clin Exp Res 2000;24:1028-35.

7 Lane J, Proude EM, Conigrave KM, et al. Nurse-provided screening and brief intervention for risky alcohol consumption by sexual health clinic patients. Sex Transm infect 2008;84:524-7.

8 Thorley N, Hettiarachchi N, Nightingale P, et al. Screening for hazardous alcohol consumption in a sexual health clinic; a service evaluation. Int J STD AIDS 2012;23:585-8.

9 Patton R, Keaney F, Brady M. Drugs, alcohol and sexual health: opportunities to influence risk behaviour. BMC Research Notes 2008;1:27.

10 Moyer A, Finney J, Swearingen C, et al. Brief interventions for alcohol problems: a meta-analytic review of controlled investigations in treatment-seeking and non-treatment-seeking. Addiction 2002;97:279-92.

11 Jenkins RJ, McAlaney J, McCambridge J. Change over time in alcohol consumption in control groups in brief intervention studies: systematic review and meta-regression study. Drug and Alcohol dependence 2009;100:107-14.

12 Baguley S. Electronic patient records and use of IT: Texting or talking? Which works better to reduce hazardous drinking by sexual health clinic attendees? Texting or Talking? Sex Transm Infect 2012;88 (Suppl 1):P112. 